\title{
DISTRIBUTED ELECTRONIC SYSTEM FOR MONITORING AND CONTROL OF A FAST SHIP PHYSICAL MODEL
}

\author{
Jose M. Girón-Sierra ${ }^{(1)}$, S. Esteban ${ }^{(1)}$, J. Recas ${ }^{(1)}$, B. De Andres-Toro( ${ }^{(1)}$, \\ E. Besada(1), J. M. De la $\mathrm{Cruz}^{(1)}$, J. M. Riola ${ }^{(2)}$
}

\begin{abstract}
This paper is related with a research on vertical motion alleviation of fast ferries. A scaled down replica of a fast ferry was built, for experimental studies in a towing tank facility. Some submerged moving actuators were added to the replica: a pair of transom flaps, lateral fins and a T-foil near the bow. Two series of experiments with waves generated in a large basin must be done. One of the series is devoted to modelling, and the second for modelbased control studies. Due to the fast motions of the experimental ship, it is not possible to attach it to a computerized carriage with instrumentation (it is part of the towing tank facility). Instead, the experimental ship must be autonomous, with all monitoring and control systems on board. Since there are six motions of the ship to be considered, the number of on-board sensors and actuators, and the complexity of control, take us to decide the design of a distributed electronic system. It is based on a central embedded PC, several microcomputer nodes, and the CANbus. The on board system interacts, using a wireless data link, with an off-shore experiment control and data processing system, with an interesting visualization performance. The purpose of the paper is to introduce this system and the associated experimental framework.
\end{abstract}

\section{KEYWORDS}

Distributed systems. Fieldbus. Ship control. Embedded systems. Monitoring and control.

\section{INTRODUCTION}

Fast ships offer evident advantages. Also they bring into attention several problems. For instance the effects of brisk motions on passengers comfort and even on ship's safety. This paper is related with a research on motion alleviation. A scaled down (1/40) replica of a fast ferry was built for experimental studies at a towing tank facility: CEHIPAR ("Canal de Experiencias Hidrodinamicas de El Pardo", Madrid, Spain). Several submerged moving actuators were added to the replica: a pair of transom flaps, lateral fins, and a T-foil. By means of control units, the actuators must move effectively to counteract the effect of each incident wave, one by one. This constitutes a problem of optimal control design.

To achieve the development of the control method, some experiments must be carried out, first to determine a mathematical model of the ship's dynamics, and second to validate the results of the control design. The experiments will be done on a basin with wavemaker. The replica must be equipped with sensors, actuators, control units and a data processing and communication system.

A distributed monitoring and control on-board system has been designed and implemented, based on the use of an embedded PC, several microcomputer nodes, and the CANbus. In addition, an off-shore system has been developed and

\footnotetext{
${ }^{1}$ Departamento A.C.Y.A., Facultad de Ciencias Físicas. Universidad Complutense de Madrid.

${ }^{2}$ Canal de Experiencias Hidrodinámicas de El Pardo (CEHIPAR).
} 
implemented for experiment programming, monitoring and control, and for off-line studies using powerful visualization features. The on-board system and the off-shore system interact through a digital wireless link.

The on-board system is subject to stringent experimental requirements, concerning weight, size , robustness and energy consumption.

The paper begins with a description of the experimental research, highlighting the peculiar aspects to be considered. Next, the paper focus on the distributed electronic on-board system. The paper continues with the off-shore system. Finally some conclusions and future work are presented.

\section{DESCRIPTION OF THE EXPERIMENTAL RESEARCH}

\subsection{Objectives}

An important shipbuilder proposed the target for this research: to alleviate the motions of a series of modern fast ferries, by means of moving submerged actuators. Some of these motions, with effects such slamming or deck wetness, can imply structural risks, mechanical fatigue, or navigation problems. Concerning passengers comfort, which is an important economical factor today, vertical motions are specifically negative. In particular, mathematical models of sea-sickness (O'Hanlon and MacCawley, 1974; Lloyd, 1998), show that this is a cumulative effect caused by vertical oscillatory accelerations with around $1 \mathrm{rad} / \mathrm{sec}$. frequency.

One of the fast ships of the series was selected, to establish a tangible case for experimental research. It is a fairly large fast ferry, with the following characteristics: $110 \mathrm{~m}$. long, 1,250 passengers, aluminium-made deep- $\mathrm{V}$ monohull, able to reach 40 knots or more. She uses powerful waterjets for propulsion and heading, having no rudder. This ferry is actually operating, between Denmark and Norway. The internal distribution of the ship, with a large market in the center, makes the passengers to be seated both sides of the ship or near the bow. These places experiment the highest vertical accelerations.

According with the technical advice of the shipbuilder company, some actuators were selected for the research: transom flaps, lateral fins and a Tfoil. The fins are of common use for roll compensation. The transom flaps are less conventional in big ships. The T-foil is placed near the bow. The flaps and the T-foil are intended mainly for pitch compensation. Few ships use a T-foil, since it may cause instability problems. All actuators are wings that should be moved (rotation) to counteract the effect of incident waves, one by one. Hydraulic cylinders can be used to move these wings.

The main problem to be solved is to design an optimal control system, to move the actuators in the most effective way, taking into account the dynamic behavior of the ship.

\subsection{Research Methodology}

There are six motions to be considered in the research. Three are translations: surge, heave and sway. Three are rotations: roll, pitch and yaw. Vertical accelerations causing sea-sickness are due to pitch, heave and roll. Mathematical models of ship motions (Fossen, 1994; Lloyd, 1998) usually consider two sets of non-coupled motions: one is formed by roll, sway and yaw, and the other by surge, heave and pitch.

The desirable scenario for control design is to have a good mathematical model of the dynamics of the plant to be controlled. It is also desirable that this model be in the form of state variables or transfer functions (when applicable).

A key fundament of this research has been the experimental support given by CEHIPAR: a towing tank facility. It is a common method in seakeeping studies to use scaled down replicas (physical models) of ships, and test them in a basin with a wavemaker system. In particular, regular waves can be generated. These waves can be considered, as an approximation, a sinusoidal input to the plant (the ship). In this way, transfer functions can be used to model the ship's dynamical behavior, for fixed conditions of heading and speed.

This research started with the simplest case, which is the vertical motions induced by head seas: only surge, heave and pitch. There is no roll, sway and yaw. A series of experiments were done to obtain data for modeling purposes, using a 1/25 scaled down replica $(4 \mathrm{~m}$. long). Control-oriented models were obtained for 20, 30 and 40 knots speed. With these models, a SIMULINK simulation tool was developed, to check the predicted performances of control designs (for instance, optimal PID, robust control methods, etc.). The best candidates for control were tested in the replica, meaning a second series of experiments. The results obtained during this initial step of the research were encouraging ( Esteban, et al. 2000, 2002; Girón-Sierra, et al. 2001). In particular, slamming and deck wetness was completely avoided, and sea sickness radically decreased (at the normal operational conditions).

Figure 1 shows a photograph of the replica in the basin, during experiments. 


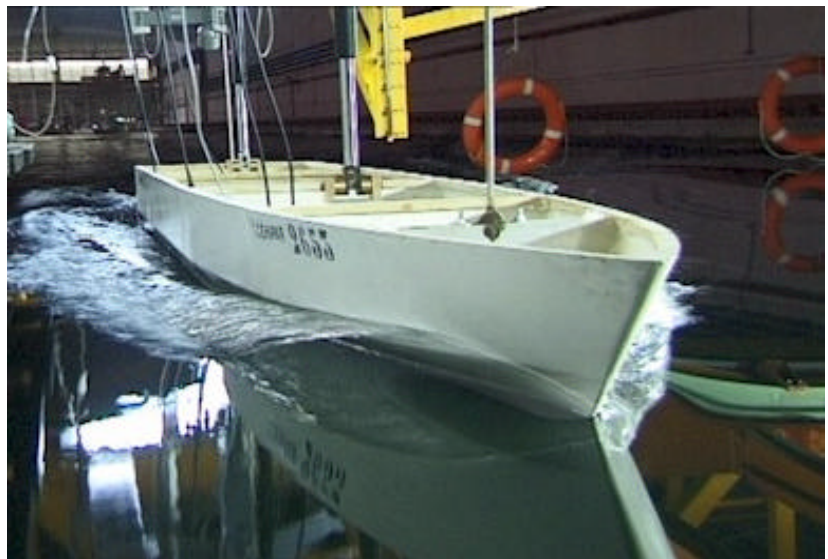

Fig. 1. Photograph of an experimental replica of the fast ship.

Along the research, regular waves are used to obtain data for modelling, and irregular waves are used to validate models and study control efficiency. The irregular waves are generated simulating ocean conditions. The studies on ocean waves present statistical mathematical models (Lloyd, 1998), with a set of predicted spectra of waves. Several sea states are typified, from very quiet to enormously rough: SSN0, SSN1, ...SSN9. In our studies, SSN4, 5 and 6 were selected, since they correspond to usual operational conditions of the ship (well, SSN6 is rough enough, with a significant wave height of 5 meters, to make advisable not to abandon the harbour).

Now the research considers the alleviation of ship's motions for any heading. The complexity of the stabilization problem increases, since all six motions of the ship must be considered. New experiments for modelling and control must be designed and run, following the path of the initial research. As will be explained below, several problems lead us to design and implement a new, smaller replica. This replica must include, on board, a fairly complex electronic system.

\subsection{Experimental Details}

The CEHIPAR facility provides two main instruments for this research: a long channel and a basin. The channel is $300 \mathrm{~m}$. long, with quiet waters. It has been used to study the forces and moments caused by the actuators wings, at several angles of attack. The CEHIPAR basin with wavemaker, is $150 \mathrm{~m}$. long (north-south), $30 \mathrm{~m}$. wide (east-west), and $5 \mathrm{~m}$. depth. It can generate waves from north to south, across $150 \mathrm{~m}$., with a front perpendicular to the north-south basin walls.

During the first studies, with head seas. The full length $(150 \mathrm{~m}$.) of the basin can be used, and the replica was towed by a Planar Motion Computerized
Carriage. All processing and control systems were in the Carriage, connected with cables to the sensors in the replica. Figure 2 shows photographs of the basin and the Carriage.

For the research starting now, any heading, with respect to waves, is considered. Suppose a heading parallel to waves, this implies to travel (east-west) only $30 \mathrm{~m}$ across the basin. Too short to obtain enough seakeeping data with the $4 \mathrm{~m}$. replica at speed equivalent to 40 knots (the equivalent is 8 knots that is $4 \mathrm{~m} / \mathrm{s}$ and so approximately 7 seconds for the $30 \mathrm{~m}$.). In consequence a smaller replica is needed. Also, it makes sense to find alternatives to the basin, for larger distances and useful data time intervals.
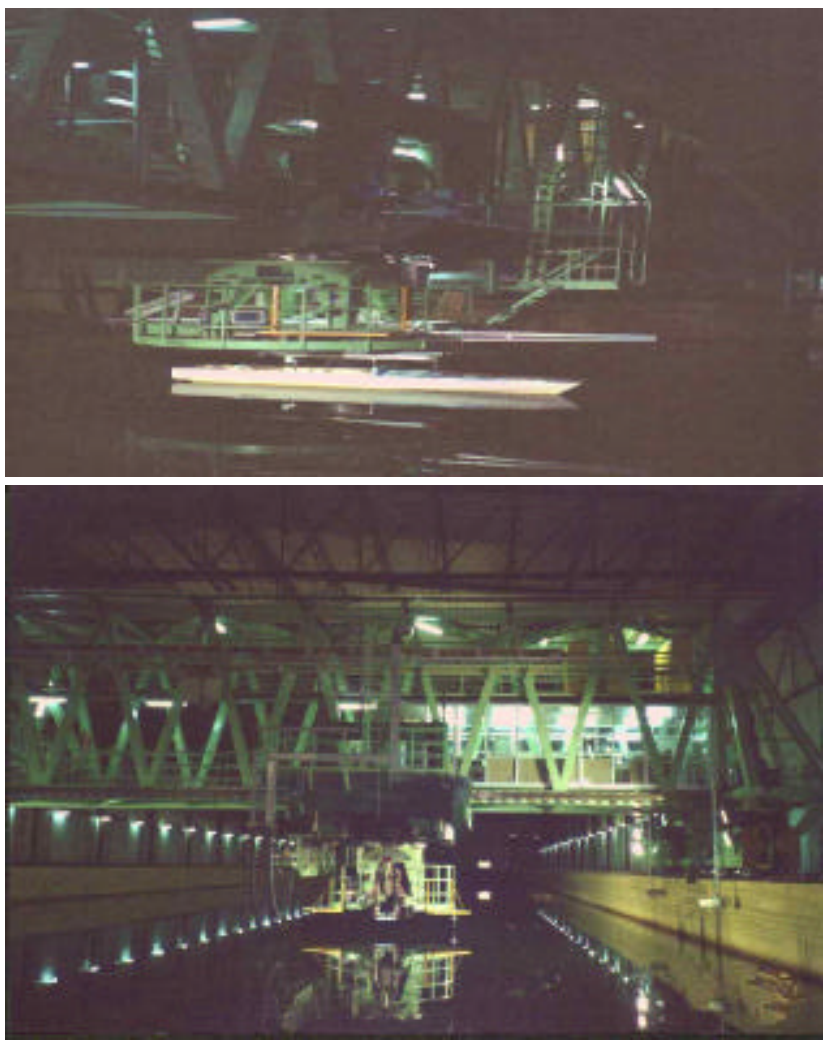

Fig. 2. Photographs of CEHIPAR basin.

There are some expected problems with actuators. For instance, compensation of roll, by moving a fin, may imply a heading deviation. Also, asymmetries in the T-foil can cause list and, again, heading difficulties. These phenomena should be studied with a free replica (not with a replica attached to the Carriage). Therefore, an autonomous replica is needed. There will be no cables connecting the replica to external instrumentation or control system.

In order to carry out the experiments, a new replica $(1 / 40)$ has been built, and a new monitoring and control electronic system, with wireless communication, has been developed. The concept of this system is modular, for "universal" use. 


\subsection{Concept of the Electronic System}

It was decided to develop a complete on board electronic system for the monitoring and control of an autonomous replica. In addition, another system has been developed for support of experiments afar from the replica (the ESS: External Support System). The missions of the on board system are the following:

- To acquire, condition and record all signals from on board sensors along experiments.

- To control the actuators of the ship.

- To transmit real-time data to the ESS.

- To transmit the complete record of data, at the end of experiments, to the ESS.

- To obey to orders given by the ESS.

The replica and the ESS communicate through radio, using digital packets. A protocol has been defined for the purposes of the experiments. The ESS can be located at the border of the basin, or perhaps in a boat (in open air experiments). It was noticed that research with autonomous naval physical models could add interesting features to the experimental facility. Therefore, the architecture of the on board system must be modular and flexible. The idea is to provide a sort of "universal" monitoring and control system, able to be applied quickly and easily to any replica.

From the side of functional structure and software, a modular concept is also useful for flexibility and fast application. The wireless communication paves the way for the use of internet. This is a powerful idea: experiments from distance.

\section{ON-BOARD DISTRIBUTED SYSTEM}

\subsection{Application Characteristics}

The decision was taken to build a new $1 / 40$ replica, the smallest size having results confidence. Since the real scale ship is aluminium made, the weight of the replica must be less than $16 \mathrm{~kg}$. This is an implementation challenge. Sophisticated techniques have been applied to build a very light hull. There are important limitations to the weight and energy consumption of the on board system. All the energy must be obtained from on board batteries (an important contribution to total weight).

The replica has two scaled down waterjets, with an additional appendage to change the jet orientation. There is no rudder. The waterjets are driven by powerful DC motors, with a maximum consumption each of 30 amps (at $6 \mathrm{~V}$ ). Scaled down flaps, T-foil and fins were added to the replica. These actuators are moved by fast servomotors. One of the sensors is an electronic compass. It was isolated by anti- magnetic film from the rest of the system, to avoid the influence of motors (which create magnetic fields).

Figure 3 depicts a diagram of main functions of the on board system. One of the blocks in the figure includes sensors for data acquisition and control. Another block is devoted to actuators handling. A third block is in charge of wireless communications. And finally there is a central block, with a low power embedded computer, for govern, coordination and data processing. Notice that there is a block to admit high priority remote control orders. The purpose of this last block is to be able to stop and recover the replica when there are problems.

Along experiments, the system measures all six motions and accelerations. Some measurements are redundant, due to a variety of reasons (safety, bias compensation, time constants, etc.).

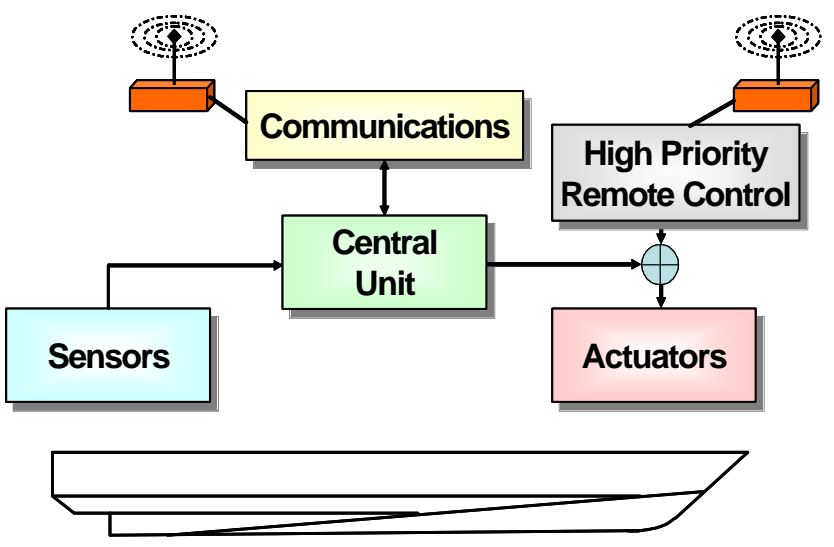

Fig. 3. Main functions of the on board system

\subsection{Wireless Communications}

The heart of the wireless communication system is a radio packet controller (RPC), from Radiometrix. It serves as a digital wireless transceiver. Physically is a small metallic module, requiring some milliamps at $5 \mathrm{v}$. The data bytes are packed as sets of 1 to 27 bytes for transmission. The module includes protocols for safe transmission. The data rate is 40 $\mathrm{kbits} / \mathrm{s}$.

At the border of the basin, or in another place far from the replica, the ESS is made with a conventional (portable) computer and a box containing another RPC and a microcontroller. Box and computer communicate via serial RS232 port.

A distribution of tasks, between replica and ESS, has been defined for normal experiments and for abnormal situations. Protocols for data monitoring and for control, along experiments and also special events, have been developed. Routines have been programmed in modular form. 
An important feature of the onboard computer is that it handles a Flash Card (like a digital camera), where several hours of experimental data, up to $1 \mathrm{~GB}$, can be stored. The bandwidth of the transceiver allows for a transmission of some important real-time data along experiments, for ESS monitoring purposes. In the end of each successful experiment, all data can be transmitted from the magnetic card to the ESS (or even, data can be obtained taking directly the card). Think about real operational conditions: it may happen that experiments take place in the sea, and it could be difficult to approach the replica to take the card: it is better a wireless transmission.

\subsection{System Architecture}

The nucleus of the on board system is an embedded PC. It has been selected according with the following criteria: small size, light weight, small energy consumption, enough computing power. After considering several alternatives, the Tern 586 Engine was chosen.

Figure 4 shows a diagram with the architecture of the on board system. It consists in seven nodes connected via CANbus. The heart of each node is a PIC 18F485 microcontroller, which includes in firmware the CANbus protocol. The embedded PC access the bus via one of these microcontrollers. The reasons for using a CANbus are related to the complexity of the system, and the long distances that can be expected for real applications on ships. This bus is well proven, and there are easy to get off-the-shelf components for building a CANbus based system.

There is only one exception about the CANbus use in the on-board system. The $A / D$ channels of the embedded PC are used for a direct, fast interaction with a Crossbow inertial unit, for 6DOF measurements. This inertial unit has $2 \mathrm{Kg}$. weight, and 1 amp. consumption.

At the stern of the ship there are two waterjets, two servos for orientation of the waterjets, two servos for reversing the water flow (for the ship to go back), and two servos to move the flaps. By means of PWM control, the speed of the motors driving the waterjets can be controlled. All these motors are controlled by two CANbus nodes.

There are three accelerometers. One is devoted to measure the worst vertical accelerations suffered by passengers (for head seas), due to pitch and heave motions. It is located near the bow. The other two are located both sides of the ship, to measure the worst vertical accelerations (for $90^{\circ}$ seas) due to roll. One of the CANbus nodes, placed near the center of the ship, is in charge of the accelerometers. It also drives, with servos, the two lateral fins.
Another CANbus node, also near the center of the ship, is used for some other sensors, such an inclinometer, a speed meter, and a wave height (relative to the waterline of the ship) sensor. There are two more CANbus nodes near the bow. One is devoted to wireless communication. The other handles an electronic digital compass, and two servos for the T-foil wings.

Part of the tasks of the microcontrollers is to simulate the dynamic characteristics of the hydraulic cylinders, which, in reality move the actuators.

The use of a CANbus imply the design of a messaging protocol, assigning i.d. numbers to each kind of message. In this case, a simple method was devised, with the embedded PC marking sampling periods, asking to sensors, and giving orders to actuators. A code module for the CANbus interaction was developed to be handled by control and monitoring programs. These control and monitoring programs are developed on conventional PC computers. Once an application program is successfully developed, the user compiles it and gets an executable code. This code is further downloaded to the embedded PC.

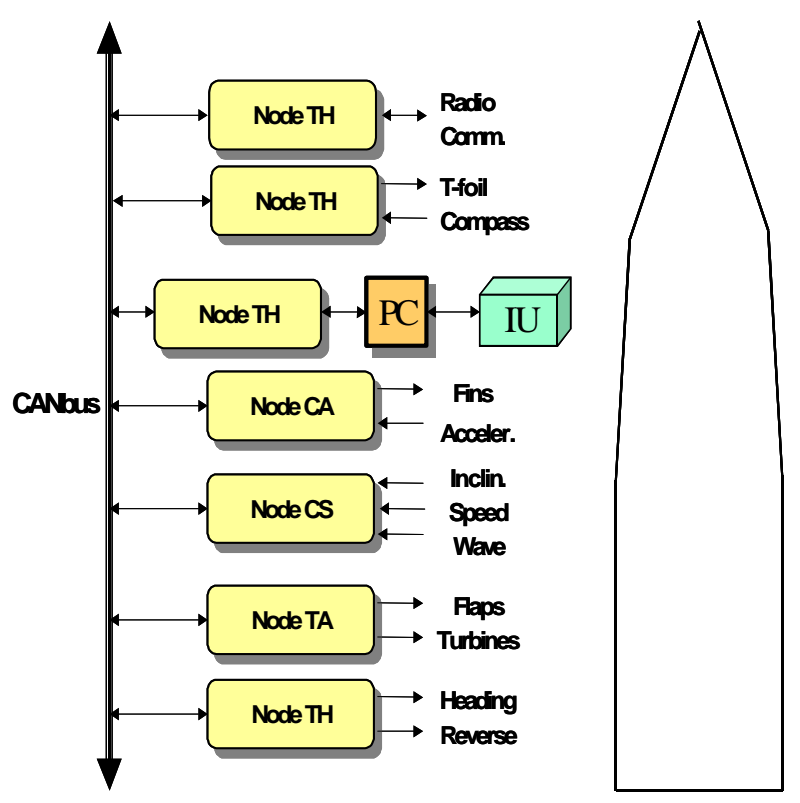

Fig. 4. Architecture of the on board system.

An advantage of the use of a fieldbus is that the several parts of the system (radio communication, servomotors, compass, etc.) can be easily distributed in different places, perhaps at relatively long distances. For instance, the compass should be placed far form magnetic fields; and radio communications should no interfere with data acquisition. 


\section{OFF-SHORE SYSTEM}

Using the powerful tools of Builder $\mathrm{C}++$, a Windowsbased visualization environment has been developed for monitoring of signals coming from the replica to the ESS. One of the reasons for the monitoring of experiments is to ensure that sensors work correctly, and the experiment is successful. Figure 5 shows a screen of the monitoring application.

A second function of the ESS is to receive the data from the replica at the end of experiments, to record them and to analyze them.

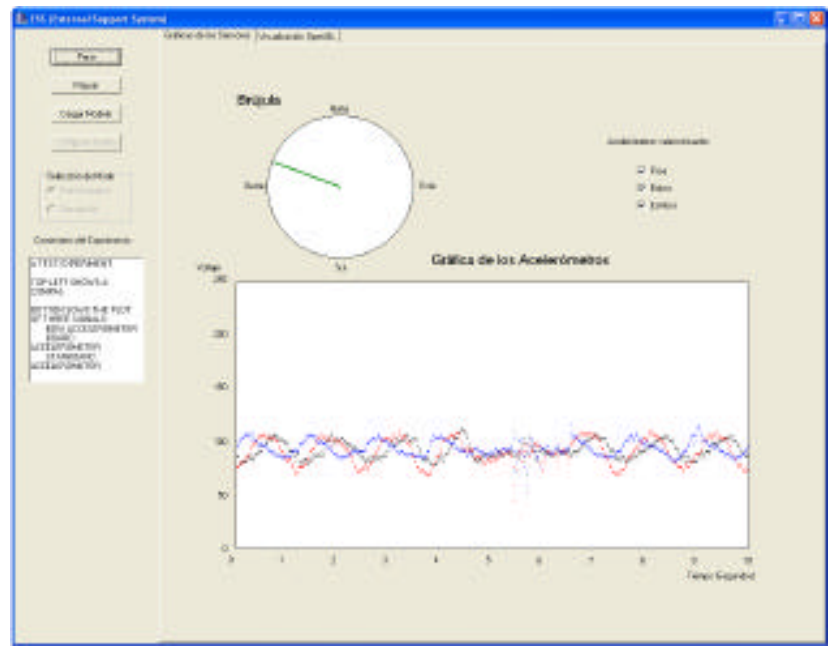

Fig. 5. A screen of the monitoring system.

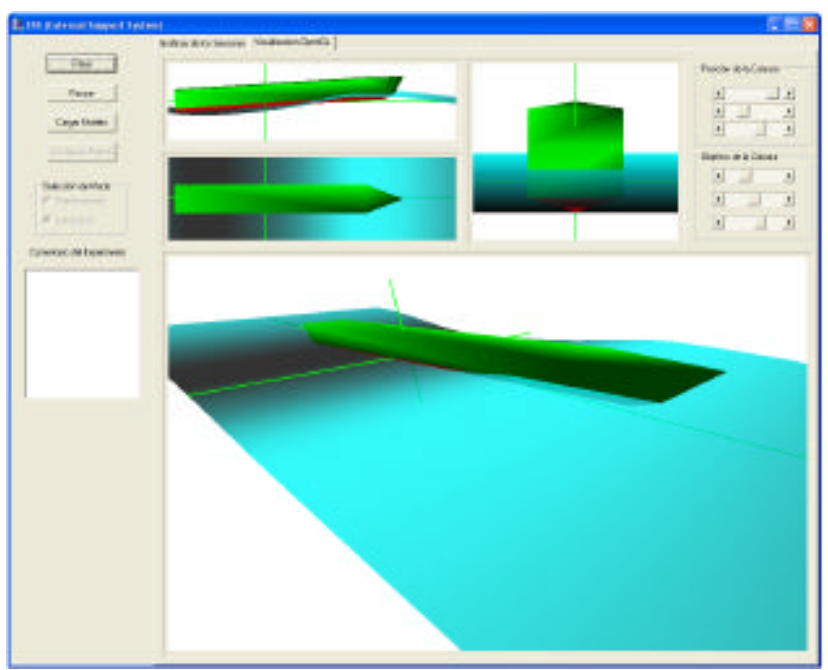

Fig.6. The 3D animated visualization system

Since it is difficult from the huge set of data to get a complete idea of what happened, a sort of dynamic reconstruction of events has been developed. Using OpenGL, a visualization of the ship with animated 3D graphics has been achieved. It is possible to handle a virtual camera, to see the ship from different points of view. This visualization can be linked to the recorded data (for example, to examine in detail slamming events). Moreover, the visualization can be also linked to the real time data during experiments, to offer a more complete view of what happens. Figure 6 shows a screen of the 3D visualization system.

The monitoring system is completed with a tool for programming manoeuvres to be done by the replica. In this way, experiments can be dynamically designed. Figure 7 shows the structure of blocks of the ESS software.

The monitoring software is based on a central nucleus, Central Manager, for coordination tasks and for data storage in a Data Pool. The rest of modules can access to the data stored in this pool. The Central Manager has two modes: Real-Time Experiment Monitoring, and Experiment Replay.

The Data Records module is a conventional file management system, at the service of the Central Manager. The Monitoring Panel is in charge of data visualizations tasks (plots). The 3-D Pane/ visualizes in animated 3-D graphics the motions of the ship. It also includes the three orthogonal projections (views) of the ship, for seakeeping studies about the six motions of the ship. The 2-D Panel is intended for trajectories and manoeuvring. It consist in a rectangle where the desired trajectory is programmed with the mouse, and where the real trajectory of the ship, obeying to orders and internal control, is displayed (to be compared with the reference trajectory). The Data Treatment Panel is in charge of several data processing functions. Experimental signals need filtering and statistical processing. Information must be extracted from signals, such the Worst Vertical Acceleration, the Motion Sickness Index, etc.

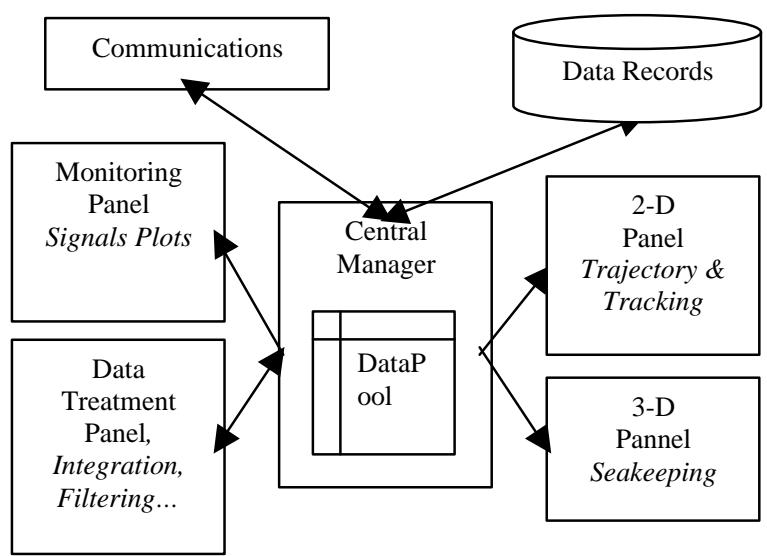

Fig.7. Software architecture of the ESS 


\section{CONCLUSIONS}

According with the new requirements of the research, that is starting to consider 6 DOF motions of a fast ferry and the use of several moving actuators, a new monitoring and control system has been developed. The purpose is to devise an experimental environment with an autonomous scaled replica, having an on board computerized system and a wireless connection to an external computer.

Relatively strict requirements of weight, size and power, made difficult to develop the on board system. However, the results obtained are encouraging. The distributed architecture, based on the CANbus, is flexible enough to be applied to other naval physical models (or even real scale ships).

\section{REFERENCES}

[1] Esteban, S., J.M. De la Cruz, J.M. Girón-Sierra, B. De Andres, J.M. Diaz and J. Aranda (2000). Fast ferry vertical acceleration reduction with active flaps and T-foil, in Proc. IFAC Intl. Symp. on Maneuvering and Control of Marine Craft, Aalborg, Denmark.

[2] Esteban, S., B. Andres-Toro, E. Besada-Portas, J.M. Girón-Sierra and J.M. de la Cruz (2002). Multiobjective control of flaps and T-foil in highspeed ships, IFAC World Congress, Barcelona.

[3] Girón-Sierra, J.M., S. Esteban, B. De Andres, J.M. Diaz and J.M. Riola (2001). Experimental study of controlled flaps and T-foil for comfort improvement of a fast ferry, in Proc. IFAC Intl. Conf. Control Applications in Marine Systems, Glasgow, U.K.

[4] Fossen, T.J. (1994), Guidance and Control of Ocean Vehicles, John Wiley.

[5] Lloyd, A.R.J.M. (1998), Seakeeping: Ship Behavior in Rough Weather, A.R.J.M. Lloyd, Gosport, Hampshire, U.K.

[6] O'Hanlon, J.F. and M.E. MacCawley (1974), Motion sickness incidence as a funtion of frequency and acceleration of vertical sinusoidal motion, Aerospace Medicine. 